\title{
Combination-Combination Hyperchaos Synchronization of Complex Memristor Oscillator System
}

\author{
Zhang Jin-E \\ School of Mathematics and Statistics, Hubei Normal University, Huangshi, Hubei, China \\ Correspondence should be addressed to Zhang Jin-E; zhang86021205@163.com
}

Received 30 March 2014; Accepted 3 May 2014; Published 29 May 2014

Academic Editor: He Huang

Copyright ( 2014 Zhang Jin-E. This is an open access article distributed under the Creative Commons Attribution License, which permits unrestricted use, distribution, and reproduction in any medium, provided the original work is properly cited.

\begin{abstract}
The combination-combination synchronization scheme is based on combination of multidrive systems and combination of multiresponse systems. In this paper, we investigate combination-combination synchronization of hyperchaotic complex memristor oscillator system. Several sufficient conditions are provided to ascertain the combination of two drive hyperchaotic complex memristor oscillator systems to synchronize the combination of two response hyperchaotic complex memristor oscillator systems. These new conditions improve and extend the existing synchronization results for memristive systems. A numerical example is given to show the feasibility of theoretical results.
\end{abstract}

\section{Introduction}

Since the characteristics of passive two-terminal memristor are reviewed, lots of emulators and macromodels have been proposed. One remarkable use for memristor is in complex memristor oscillator systems, where nonlinear oscillators from Chua's oscillators are replaced by using memristors [110]. The complex behaviors for such systems were analyzed in $[1-5,8-10]$. When nonlinear memristor is applied to electronic device, the behavior of the new-type device becomes complicated and difficult to predict due to the unique nonlinear mechanism of memristor [11-14]. However, to apply to the practical engineering, its electrical characteristics must be able to be fully understood. So it is vital to understand the characteristics and behaviors of memristive devices. In [1], the transient chaos was studied for a smooth fluxcontrolled complex memristor oscillator system. In [2], the local and global behavior of the basic oscillator on complex memristor oscillator system was investigated. Itoh and Chua [3] discussed some interesting oscillation properties and rich nonlinear dynamics of several complex memristor oscillator systems from Chua's oscillators. Talukdar et al. [7] reported the nonlinear dynamics of three memristor-based phase shift oscillators. As we all know, the electrical characteristics of complex memristor oscillator system can vary depending upon its circuit structure. The internal behavior of high order (fifth-order or more) complex memristor oscillator system still is not being analyzed [9, 10]. In high order complex memristor oscillator system, there are still many unknown fields waiting for us to exploit them. Specifically, the current studies about complex memristor oscillator system have mostly remained at the basic chaotic dynamic theory. Compared with common chaos with only one positive Lyapunov exponent, hyperchaos with more than one positive Lyapunov exponents can exhibit multidirectional expansion and extremely complex behaviors. Can we design high order complex memristor oscillator system to achieve the hyperchaotic effect? If this is really so, many different applications might be proposed, such as ultradense nonvolatile memory and high-performance secure communication $[9,10]$.

The topic of chaos synchronization of oscillator systems is extensively investigated because of possible relevance to information encryption [8-11, 15-28]. Various kinds of synchronization laws have been obtained, for example, complete synchronization $[8,11,15-18]$, compound synchronization [10], antisynchronization [19-21], phase synchronization [22, 23], lag synchronization [24-26], projective synchronization $[10,27]$, and combination synchronization $[9,28]$. However, in the existing literature, most of synchronization laws are based on one drive system and one response system. In 
this way, when chaos synchronization is applied to secure communication, the information signal is transmitted by only one chaotic system, which is not suitable for very highperformance secure communication. Can the information signal be transmitted by the combination of multiple chaotic systems? If we can successfully provide some ways to do this, then the transmitted signals will be more complex and unpredictable, and thereby the security of communication can be effectively enhanced. Thus, a theoretical question is whether we can design a synchronization scheme on the combination of multidrive systems and combination of multiresponse systems. Such a combination design may help to improve the secrecy in communications $[9,28]$.

Motivated by the above discussions, in this paper, firstly, a new hyperchaotic complex memristor oscillator system is introduced and studied. Secondly, we bring in the scheme of combination-combination synchronization and establish some synchronization criteria. Roughly stated, the main advantages of this paper include the following three points.

(1) Hyperchaotic complex memristor oscillator system is systematically investigated. Hyperchaos is often considered better than common chaos in many engineering fields. Theoretically, hyperchaotic complex memristor oscillator system might improve the security of chaotic communication system.

(2) The synchronization control between combination of two drive hyperchaotic complex memristor oscillator systems and combination of two response hyperchaotic complex memristor oscillator systems in drive-response coupled system is investigated. The generalization of synchronization scheme will provide a wider scope for the designs and applications of memristive system.

(3) The proposed scheme of combination-combination synchronization in this paper can be applied to the general nonlinear systems.

The rest of this paper is arranged as follows. Section 2 describes some preliminaries and problem formulation. Section 3 derives some sufficient conditions on combinationcombination synchronization for hyperchaotic complex memristor oscillator system and then presents a numerical example to demonstrate the validity of the theoretical results. Section 4 concludes the paper with some remarks.

\section{Preliminaries}

Consider a fifth-order complex memristor oscillator system with its dynamics described by the following ordinary differential equations:

$$
\begin{aligned}
& \dot{v}_{1}(t)=\frac{1}{C_{1}} \ell_{1}(t)-\frac{1}{C_{1}} W_{1}(\varphi(t)) v_{1}(t), \\
& \dot{v}_{2}(t)=-\frac{1}{C_{2}} \ell_{1}(t)+\frac{1}{C_{2}} \ell_{2}(t),
\end{aligned}
$$

$$
\begin{aligned}
& \dot{\ell}_{1}(t)=\frac{1}{L_{1}} v_{2}(t)-\frac{1}{L_{1}} v_{1}(t)-\frac{R}{L_{1}} \ell_{1}(t), \\
& \dot{\ell}_{2}(t)=-\frac{1}{L_{2}} v_{2}(t), \\
& \dot{\varphi}(t)=v_{1}(t),
\end{aligned}
$$

where $v_{1}(t)$ and $v_{2}(t)$ denote voltages, $\ell_{1}(t)$ and $\ell_{2}(t)$ represent currents, $C_{1}$ and $C_{2}$ denote capacitors, $L_{1}$ and $L_{2}$ represent inductors, $W(\varphi(t))$ is memductance function, and $\varphi(t)$ and $R$ are magnetic flux and resistor, respectively.

Similarly as in $[1,9,10]$, a cubic memristor is chosen; then

$$
W(\varphi(t))=a+3 b \varphi(t)^{2},
$$

where $a$ and $b$ are parameters.

From (1) and (2),

$$
\begin{aligned}
& \dot{v}_{1}(t)=\frac{1}{C_{1}} \ell_{1}(t)-\frac{a}{C_{1}} v_{1}(t)-\frac{3 b}{C_{1}} v_{1}(t) \varphi(t)^{2}, \\
& \dot{v}_{2}(t)=-\frac{1}{C_{2}} \ell_{1}(t)+\frac{1}{C_{2}} \ell_{2}(t), \\
& \dot{\ell}_{1}(t)=\frac{1}{L_{1}} v_{2}(t)-\frac{1}{L_{1}} v_{1}(t)-\frac{R}{L_{1}} \ell_{1}(t), \\
& \dot{\ell}_{2}(t)=-\frac{1}{L_{2}} v_{2}(t) \\
& \dot{\varphi}(t)=v_{1}(t) .
\end{aligned}
$$

Let $x_{11}(t)=v_{1}(t), x_{12}(t)=v_{2}(t), x_{13}(t)=\ell_{1}(t), x_{14}(t)=$ $\ell_{2}(t), x_{15}(t)=\varphi(t), \alpha_{1}=1 / C_{1}, \alpha_{2}=a / C_{1}, \alpha_{3}=3 b / C_{1}$, $\alpha_{4}=1 / C_{2}, \alpha_{5}=1 / L_{1}, \alpha_{6}=R / L_{1}, \alpha_{7}=1 / L_{2}$; then (3) can be rewritten as

$$
\begin{aligned}
& \dot{x}_{11}=\alpha_{1} x_{13}-\alpha_{2} x_{11}-\alpha_{3} x_{11} x_{15}^{2}, \\
& \dot{x}_{12}=-\alpha_{4} x_{13}+\alpha_{4} x_{14}, \\
& \dot{x}_{13}=\alpha_{5} x_{12}-\alpha_{5} x_{11}-\alpha_{6} x_{13}, \\
& \dot{x}_{14}=-\alpha_{7} x_{12}, \\
& \dot{x}_{15}=x_{11} .
\end{aligned}
$$

Let parameters $\alpha_{1}=9, \alpha_{2}=-10.8, \alpha_{3}=10.8$, $\alpha_{4}=1, \alpha_{5}=30, \alpha_{6}=30, \alpha_{7}=15$, the initial state $x_{11}(0)=0.01, x_{12}(0)=0.01, x_{13}(0)=0.01, x_{14}(0)=0.01$, $x_{15}(0)=0.01$, by mean of computer program with MATLAB, the corresponding Lyapunov exponents of system (4) are $0.279403,0.023752,-0.038779,-7.391447,-16.291929$. The numerical result is shown in Figure 1, where the first two Lyapunov exponents are positive. Clearly, it implies that memristor oscillator system (4) is hyperchaotic. Figure 2 describes the hyperchaotic attractors.

Many efforts have been paid in recent years to develop efficient circuit implementation for the generation of hyperchaotic memristor oscillator system. However, to the best of our knowledge, up to now, the hyperchaotic memristor 


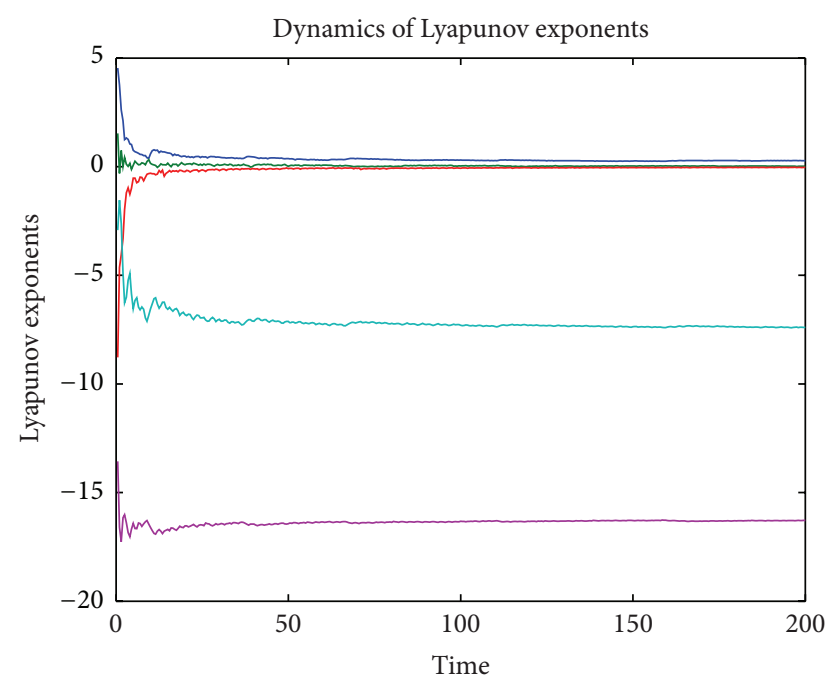

Figure 1: Dynamics of Lyapunov exponents from memristor oscillator system (4).

oscillator system is still very unusual. Thus, hyperchaotic memristor oscillator system (4) is important for our understanding of hyperchaotic memristive devices.

In the following, we end this section with the scheme of combination-combination synchronization, which will be used in subsequent section.

In engineering field, generally, combination-combination synchronization is based on multiple drive systems and multiple response systems. In many engineering applications and hardware implementations, combination-combination synchronization constituting of two drive systems and two response systems is usually considered. Figure 3 describes a schematic diagram of combination-combination synchronization scheme. In fact, combination-combination synchronization has its unique physical interpretation. For example, in Figure 3, the combination of two drive systems generates the resultant signal; then the combination of two response systems tracks the resultant signal.

Next, we give specific mathematical descriptions of combination-combination synchronization scheme.

Consider the drive system:

$$
\dot{\chi}_{1}=f_{1}\left(\chi_{1}\right) .
$$

The other drive system is given by

$$
\dot{\chi}_{2}=f_{2}\left(\chi_{2}\right) \text {, }
$$

and two response systems are described by

$$
\begin{aligned}
& \dot{\chi}_{3}=f_{3}\left(\chi_{3}\right)+u, \\
& \dot{\chi}_{4}=f_{4}\left(\chi_{4}\right)+u^{*},
\end{aligned}
$$

where state vectors $\chi_{1}=\left(\chi_{11}, \chi_{12}, \ldots, \chi_{1 n}\right)^{T}, \chi_{2}=\left(\chi_{21}\right.$, $\left.\chi_{22}, \ldots, \chi_{2 n}\right)^{T}, \chi_{3}=\left(\chi_{31}, \chi_{32}, \ldots, \chi_{3 n}\right)^{T}$, and $\chi_{4}=\left(\chi_{41}, \chi_{42}\right.$, $\left.\ldots, \chi_{4 n}\right)^{T}$ and vector functions $f_{1}(\cdot), f_{2}(\cdot), f_{3}(\cdot), f_{4}(\cdot)$ : $\Re^{n} \longrightarrow \Re^{n}, u=\left(u_{1}, u_{2}, \ldots, u_{n}\right)^{T}, u^{*}=\left(u_{1}^{*}, u_{2}^{*}, \ldots, u_{n}^{*}\right)^{T}:$
$\mathfrak{R}^{n} \times \mathfrak{R}^{n} \times \cdots \times \mathfrak{R}^{n} \longrightarrow \mathfrak{R}^{n}$ are the appropriate control inputs that will be designed in order to obtain a certain control objective.

Definition 1. The drive systems (5) and (6) are realized combination-combination synchronization with the response systems (7) if there exist $n$-dimensional constant diagonal matrices $A_{1}, A_{2}, A_{3} \neq 0$, and $A_{4} \neq 0$ such that

$$
\lim _{t \rightarrow+\infty}\|e\|=\lim _{t \rightarrow+\infty}\left\|A_{1} X_{1}+A_{2} X_{2}-A_{3} X_{3}-A_{4} X_{4}\right\|=0,
$$

where $\|\cdot\|$ is vector norm, $e=\left(e_{1}, e_{2}, \ldots, e_{n}\right)^{T}$ is the synchronization error vector, $X_{1}=\operatorname{diag}\left(\chi_{11}, \chi_{12}, \ldots, \chi_{1 n}\right)$, $X_{2}=\operatorname{diag}\left(\chi_{21}, \chi_{22}, \ldots, \chi_{2 n}\right), X_{3}=\operatorname{diag}\left(\chi_{31}, \chi_{32}, \ldots, \chi_{3 n}\right)$, and $X_{4}=\operatorname{diag}\left(\chi_{41}, \chi_{42}, \ldots, \chi_{4 n}\right)$.

Remark 2. As stated earlier, according to Definition 1, the physical implication of combination-combination synchronization is rather intuitionistic. The resultant signal of the combination of two drive systems (5) and (6) is tracked by the synthetic signal of the combination of two response systems (7).

Remark 3. In [28], Sun et al. apply combination-combination design to study the synchronization control of some classical chaotic systems. Generally speaking, this synchronization method for chaotic system is quite novel.

Remark 4. In Definition 1, matrices $A_{1}, A_{2}, A_{3}$, and $A_{4}$ are often called the scaling matrices. It is not hard to find that the scheme of combination-combination synchronization in Definition 1 contains the combination of two drive systems and the combination of two response systems. Moreover, one advantage of combination-combination synchronization is that the drive systems or the response systems can be completely identical or different.

Remark 5. The scheme of combination-combination synchronization improves and generalizes some existing synchronization schemes. When scaling matrices $A_{3}=0$ or $A_{4}=0$, the combination-combination synchronization will degrade into combination synchronization. When scaling matrices $A_{1}=A_{3}=0$ or $A_{1}=A_{4}=0$ or $A_{2}=A_{3}=0$ or $A_{2}=A_{4}=0$, the combination-combination synchronization will be reduced to complete synchronization. When scaling matrices $A_{1}=A_{2}=A_{3}=0$ or $A_{1}=A_{2}=A_{4}=0$, the combination-combination synchronization will change into chaos control.

\section{Synchronization Criteria}

In this section, we will devote to investigate combinationcombination synchronization of hyperchaotic complex memristor oscillator system. 


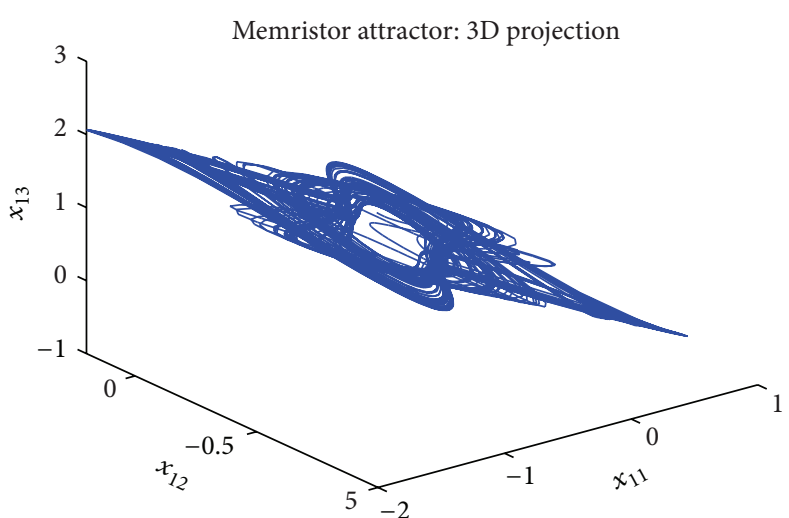

(a)

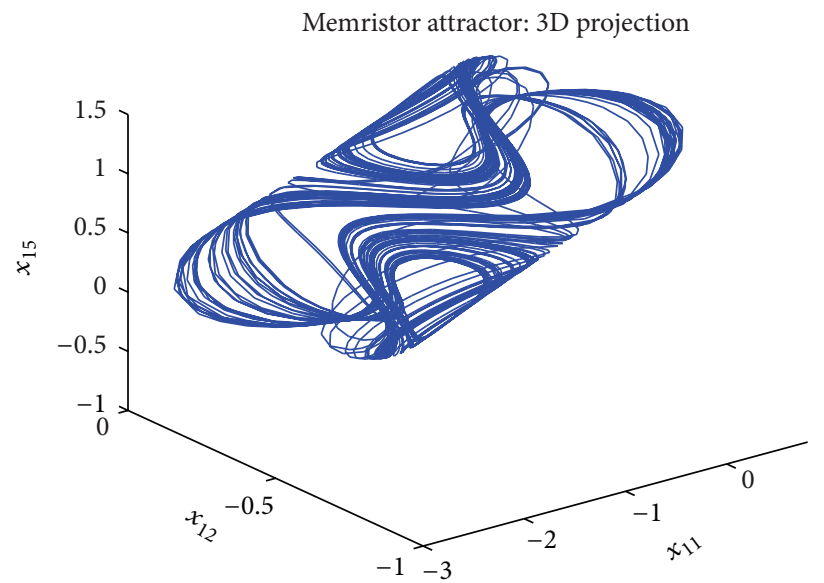

(c)

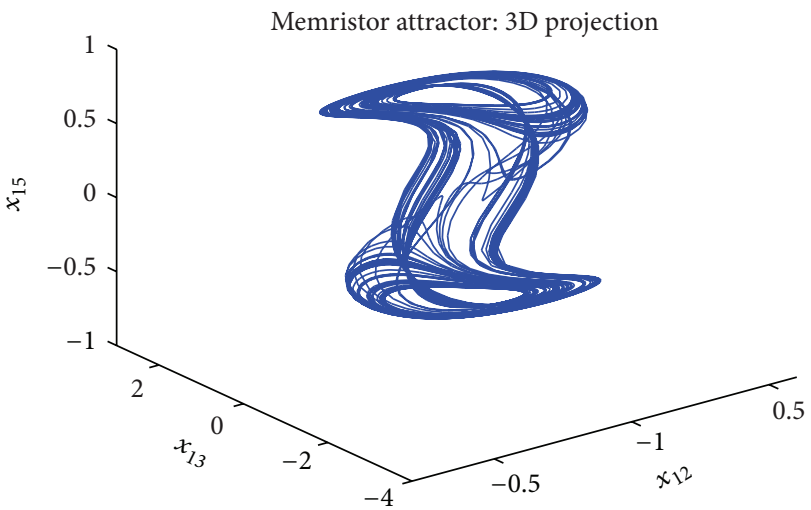

(e)

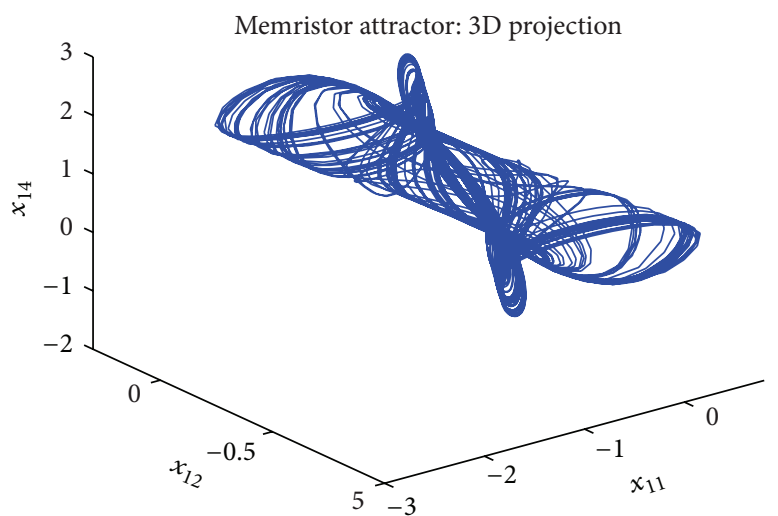

(b)

Memristor attractor: 3D projection

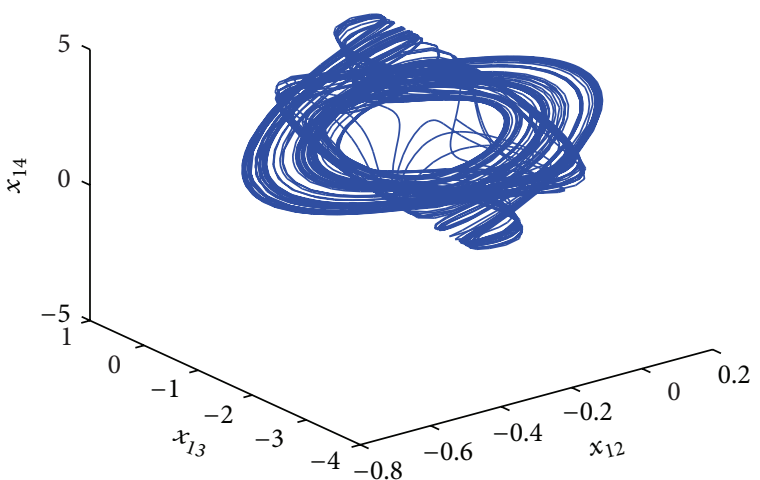

(d)

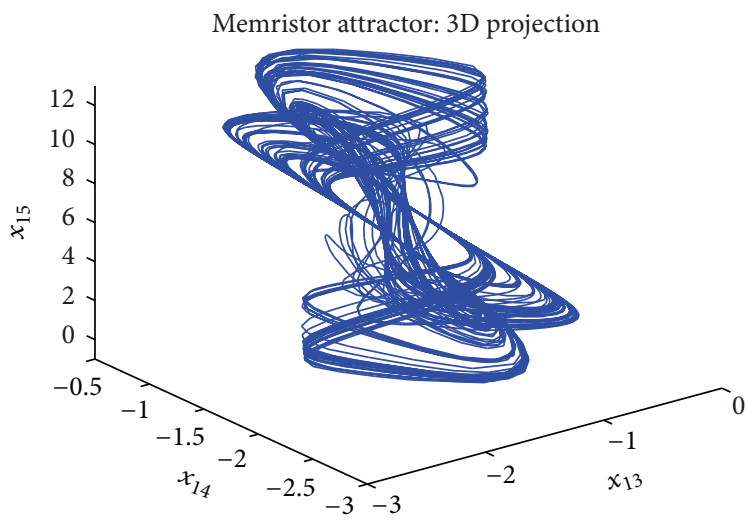

(f)

FIGURE 2: 3D projections of the hyperchaotic attractor from memristor oscillator system (4).

Corresponding to (4), the other drive system is given as

$$
\begin{aligned}
& \dot{x}_{21}=\beta_{1} x_{23}-\beta_{2} x_{21}-\beta_{3} x_{21} x_{25}^{2}, \\
& \dot{x}_{22}=-\beta_{4} x_{23}+\beta_{4} x_{24}, \\
& \dot{x}_{23}=\beta_{5} x_{22}-\beta_{5} x_{21}-\beta_{6} x_{23}, \\
& \dot{x}_{24}=-\beta_{7} x_{22}, \\
& \dot{x}_{25}=x_{21},
\end{aligned}
$$

and two response systems are described as follows:

$$
\begin{aligned}
& \dot{x}_{31}=\gamma_{1} x_{33}-\gamma_{2} x_{31}-\gamma_{3} x_{31} x_{35}^{2}+u_{1}, \\
& \dot{x}_{32}=-\gamma_{4} x_{33}+\gamma_{4} x_{34}+u_{2}, \\
& \dot{x}_{33}=\gamma_{5} x_{32}-\gamma_{5} x_{31}-\gamma_{6} x_{33}+u_{3}, \\
& \dot{x}_{34}=-\gamma_{7} x_{32}+u_{4}, \\
& \dot{x}_{35}=x_{31}+u_{5},
\end{aligned}
$$




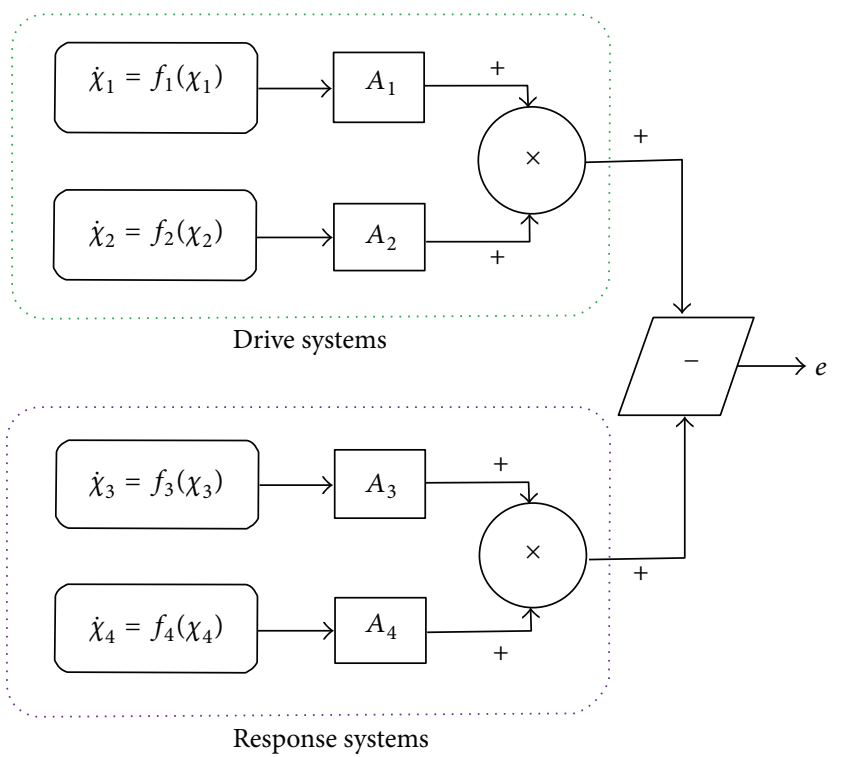

FIGURE 3: The physical realization of combination-combination synchronization scheme.

$$
\begin{aligned}
& \dot{x}_{41}=\eta_{1} x_{43}-\eta_{2} x_{41}-\eta_{3} x_{41} x_{45}^{2}+u_{1}^{*}, \\
& \dot{x}_{42}=-\eta_{4} x_{43}+\eta_{4} x_{44}+u_{2}^{*}, \\
& \dot{x}_{43}=\eta_{5} x_{42}-\eta_{5} x_{41}-\eta_{6} x_{43}+u_{3}^{*}, \\
& \dot{x}_{44}=-\eta_{7} x_{42}+u_{4}^{*}, \\
& \dot{x}_{45}=x_{41}+u_{5}^{*},
\end{aligned}
$$

where $\beta_{1}, \beta_{2}, \beta_{3}, \beta_{4}, \beta_{5}, \beta_{6}, \beta_{7}, \gamma_{1}, \gamma_{2}, \gamma_{3}, \gamma_{4}, \gamma_{5}, \gamma_{6}, \gamma_{7}, \eta_{1}, \eta_{2}$, $\eta_{3}, \eta_{4}, \eta_{5}, \eta_{6}$, and $\eta_{7}$ are parameters and $u_{1}, u_{2}, u_{3}, u_{4}, u_{5}, u_{1}^{*}$, $u_{2}^{*}, u_{3}^{*}, u_{4}^{*}$, and $u_{5}^{*}$ are the control inputs to be designed.

In our synchronization scheme, let

$$
\begin{aligned}
& A_{1}=\operatorname{diag}\left(a_{11}, a_{12}, a_{13}, a_{14}, a_{15}\right), \\
& A_{2}=\operatorname{diag}\left(a_{21}, a_{22}, a_{23}, a_{24}, a_{25}\right), \\
& A_{3}=\operatorname{diag}\left(a_{31}, a_{32}, a_{33}, a_{34}, a_{35}\right), \\
& A_{4}=\operatorname{diag}\left(a_{41}, a_{42}, a_{43}, a_{44}, a_{45}\right) .
\end{aligned}
$$

Thus,

$$
\begin{aligned}
& e_{1}=a_{11} x_{11}+a_{21} x_{21}-a_{31} x_{31}-a_{41} x_{41}, \\
& e_{2}=a_{12} x_{12}+a_{22} x_{22}-a_{32} x_{32}-a_{42} x_{42}, \\
& e_{3}=a_{13} x_{13}+a_{23} x_{23}-a_{33} x_{33}-a_{43} x_{43}, \\
& e_{4}=a_{14} x_{14}+a_{24} x_{24}-a_{34} x_{34}-a_{44} x_{44} \\
& e_{5}=a_{15} x_{15}+a_{25} x_{25}-a_{35} x_{35}-a_{45} x_{45}
\end{aligned}
$$

Obviously, we have

$$
\begin{aligned}
& \dot{e}_{1}=a_{11} \dot{x}_{11}+a_{21} \dot{x}_{21}-a_{31} \dot{x}_{31}-a_{41} \dot{x}_{41}, \\
& \dot{e}_{2}=a_{12} \dot{x}_{12}+a_{22} \dot{x}_{22}-a_{32} \dot{x}_{32}-a_{42} \dot{x}_{42}, \\
& \dot{e}_{3}=a_{13} \dot{x}_{13}+a_{23} \dot{x}_{23}-a_{33} \dot{x}_{33}-a_{43} \dot{x}_{43}, \\
& \dot{e}_{4}=a_{14} \dot{x}_{14}+a_{24} \dot{x}_{24}-a_{34} \dot{x}_{34}-a_{44} \dot{x}_{44}, \\
& \dot{e}_{5}=a_{15} \dot{x}_{15}+a_{25} \dot{x}_{25}-a_{35} \dot{x}_{35}-a_{45} \dot{x}_{45} .
\end{aligned}
$$

Combining with (4) and (9)-(11), then the synchronization error system (14) can be transformed into the following form:

$$
\begin{aligned}
& \dot{e}_{1}= a_{11}\left(\alpha_{1} x_{13}-\alpha_{2} x_{11}-\alpha_{3} x_{11} x_{15}^{2}\right) \\
&+a_{21}\left(\beta_{1} x_{23}-\beta_{2} x_{21}-\beta_{3} x_{21} x_{25}^{2}\right) \\
&-a_{31}\left(\gamma_{1} x_{33}-\gamma_{2} x_{31}-\gamma_{3} x_{31} x_{35}^{2}+u_{1}\right) \\
&-a_{41}\left(\eta_{1} x_{43}-\eta_{2} x_{41}-\eta_{3} x_{41} x_{45}^{2}+u_{1}^{*}\right), \\
& \dot{e}_{2}=a_{12}(\left.-\alpha_{4} x_{13}+\alpha_{4} x_{14}\right)+a_{22}\left(-\beta_{4} x_{23}+\beta_{4} x_{24}\right) \\
&- a_{32}\left(-\gamma_{4} x_{33}+\gamma_{4} x_{34}+u_{2}\right) \\
&- a_{42}\left(-\eta_{4} x_{43}+\eta_{4} x_{44}+u_{2}^{*}\right), \\
& \dot{e}_{3}= a_{13}\left(\alpha_{5} x_{12}-\alpha_{5} x_{11}-\alpha_{6} x_{13}\right) \\
&+a_{23}\left(\beta_{5} x_{22}-\beta_{5} x_{21}-\beta_{6} x_{23}\right) \\
&-a_{33}\left(\gamma_{5} x_{32}-\gamma_{5} x_{31}-\gamma_{6} x_{33}+u_{3}\right) \\
&-a_{43}\left(\eta_{5} x_{42}-\eta_{5} x_{41}-\eta_{6} x_{43}+u_{3}^{*}\right), \\
& \dot{e}_{5}=a_{15} x_{11}+a_{25} x_{21}-a_{35}\left(x_{31}+u_{5}\right)-a_{45}\left(x_{41}+u_{5}^{*}\right) . \\
& \dot{e}_{4}=a_{14}\left(-\alpha_{7} x_{12}\right)+a_{24}\left(-\beta_{7} x_{22}\right)-a_{34}\left(-\gamma_{7} x_{32}+u_{4}\right) \\
&-a_{44}\left(-\eta_{7} x_{42}+u_{4}^{*}\right),
\end{aligned}
$$

\section{Denote}

$$
\begin{aligned}
& U_{1}=a_{31} u_{1}+a_{41} u_{1}^{*}, \\
& U_{2}=a_{32} u_{2}+a_{42} u_{2}^{*}, \\
& U_{3}=a_{33} u_{3}+a_{43} u_{3}^{*}, \\
& U_{4}=a_{34} u_{4}+a_{44} u_{4}^{*}, \\
& U_{5}=a_{35} u_{5}+a_{45} u_{5}^{*} .
\end{aligned}
$$


Then (15) can be rewritten as

$$
\begin{aligned}
& \dot{e}_{1}= a_{11}\left(\alpha_{1} x_{13}-\alpha_{2} x_{11}-\alpha_{3} x_{11} x_{15}^{2}\right) \\
&+a_{21}\left(\beta_{1} x_{23}-\beta_{2} x_{21}-\beta_{3} x_{21} x_{25}^{2}\right) \\
&-a_{31}\left(\gamma_{1} x_{33}-\gamma_{2} x_{31}-\gamma_{3} x_{31} x_{35}^{2}\right) \\
&-a_{41}\left(\eta_{1} x_{43}-\eta_{2} x_{41}-\eta_{3} x_{41} x_{45}^{2}\right)-U_{1}, \\
& \dot{e}_{2}=a_{12}(\left.-\alpha_{4} x_{13}+\alpha_{4} x_{14}\right)+a_{22}\left(-\beta_{4} x_{23}+\beta_{4} x_{24}\right) \\
&- a_{32}\left(-\gamma_{4} x_{33}+\gamma_{4} x_{34}\right) \\
&- a_{42}\left(-\eta_{4} x_{43}+\eta_{4} x_{44}\right)-U_{2}, \\
& \dot{e}_{3}= a_{13}\left(\alpha_{5} x_{12}-\alpha_{5} x_{11}-\alpha_{6} x_{13}\right) \\
&+a_{23}\left(\beta_{5} x_{22}-\beta_{5} x_{21}-\beta_{6} x_{23}\right) \\
&-a_{33}\left(\gamma_{5} x_{32}-\gamma_{5} x_{31}-\gamma_{6} x_{33}\right) \\
&-a_{43}\left(\eta_{5} x_{42}-\eta_{5} x_{41}-\eta_{6} x_{43}\right)-U_{3} \\
& \dot{e}_{4}=-a_{14} \alpha_{7} x_{12}-a_{24} \beta_{7} x_{22}+a_{34} \gamma_{7} x_{32}+a_{44} \eta_{7} x_{42}-U_{4}, \\
& \dot{e}_{5}=a_{15} x_{11}+a_{25} x_{21}-a_{35} x_{31}-a_{45} x_{41}-U_{5} .
\end{aligned}
$$

In fact, (16) is called the controller to be designed.

Theorem 6. If the control law is chosen as follows:

$$
\begin{aligned}
U_{1}= & a_{11}\left[\alpha_{1} x_{13}+\left(1-\alpha_{2}\right) x_{11}-\alpha_{3} x_{11} x_{15}^{2}\right] \\
& +a_{21}\left[\beta_{1} x_{23}+\left(1-\beta_{2}\right) x_{21}-\beta_{3} x_{21} x_{25}^{2}\right] \\
& -a_{31}\left[\gamma_{1} x_{33}+\left(1-\gamma_{2}\right) x_{31}-\gamma_{3} x_{31} x_{35}^{2}\right] \\
& -a_{41}\left[\eta_{1} x_{43}+\left(1-\eta_{2}\right) x_{41}-\eta_{3} x_{41} x_{45}^{2}\right] \\
& +\left(a_{12} x_{12}+a_{22} x_{22}-a_{32} x_{32}-a_{42} x_{42}\right) \\
U_{2}= & a_{12}\left(x_{12}-\alpha_{4} x_{13}+\alpha_{4} x_{14}\right) \\
& +a_{22}\left(x_{22}-\beta_{4} x_{23}+\beta_{4} x_{24}\right) \\
& -a_{32}\left(x_{32}-\gamma_{4} x_{33}+\gamma_{4} x_{34}\right) \\
& -a_{42}\left(x_{42}-\eta_{4} x_{43}+\eta_{4} x_{44}\right) \\
& -\left(a_{11} x_{11}+a_{21} x_{21}-a_{31} x_{31}-a_{41} x_{41}\right) \\
& +\left(a_{13} x_{13}+a_{23} x_{23}-a_{33} x_{33}-a_{43} x_{43}\right) \\
U_{3}= & a_{13}\left[\alpha_{5} x_{12}-\alpha_{5} x_{11}+\left(1-\alpha_{6}\right) x_{13}\right] \\
& +a_{23}\left[\beta_{5} x_{22}-\beta_{5} x_{21}+\left(1-\beta_{6}\right) x_{23}\right] \\
& -a_{33}\left[\gamma_{5} x_{32}-\gamma_{5} x_{31}+\left(1-\gamma_{6}\right) x_{33}\right] \\
&
\end{aligned}
$$

$$
\begin{aligned}
& -a_{43}\left[\eta_{5} x_{42}-\eta_{5} x_{41}+\left(1-\eta_{6}\right) x_{43}\right] \\
& -\left(a_{12} x_{12}+a_{22} x_{22}-a_{32} x_{32}-a_{42} x_{42}\right) \\
& +\left(a_{14} x_{14}+a_{24} x_{24}-a_{34} x_{34}-a_{44} x_{44}\right), \\
U_{4}= & -\left(a_{13} x_{13}+a_{23} x_{23}-a_{33} x_{33}-a_{43} x_{43}\right) \\
& +a_{14}\left(x_{14}-\alpha_{7} x_{12}\right)+a_{24}\left(x_{24}-\beta_{7} x_{22}\right) \\
& -a_{34}\left(x_{34}-\gamma_{7} x_{32}\right)-a_{44}\left(x_{44}-\eta_{7} x_{42}\right) \\
& +\left(a_{15} x_{15}+a_{25} x_{25}-a_{35} x_{35}-a_{45} x_{45}\right), \\
U_{5}= & a_{15}\left(x_{11}+x_{15}\right)+a_{25}\left(x_{21}+x_{25}\right) \\
& -a_{35}\left(x_{31}+x_{35}\right)-a_{45}\left(x_{41}+x_{45}\right) \\
& -\left(a_{14} x_{14}+a_{24} x_{24}-a_{34} x_{34}-a_{44} x_{44}\right),
\end{aligned}
$$

then the drive systems (4) and (9) will achieve combinationcombination synchronization with the response systems (10) and (11).

Proof. Consider the following Lyapunov function:

$$
\begin{aligned}
V(e(t)) & =V\left(e_{1}, e_{2}, e_{3}, e_{4}, e_{5}\right) \\
& =\frac{1}{2}\left(e_{1}^{2}+e_{2}^{2}+e_{3}^{2}+e_{4}^{2}+e_{5}^{2}\right) .
\end{aligned}
$$

Calculating the upper right Dini derivative of $V$ along the trajectory of (17), then

$$
\begin{aligned}
D^{+} V=e_{1} \dot{e}_{1} & +e_{2} \dot{e}_{2}+e_{3} \dot{e}_{3}+e_{4} \dot{e}_{4}+e_{5} \dot{e}_{5} \\
=e_{1}\left[a_{11}\left(\alpha_{1} x_{13}-\alpha_{2} x_{11}-\alpha_{3} x_{11} x_{15}^{2}\right)\right. & \\
+ & a_{21}\left(\beta_{1} x_{23}-\beta_{2} x_{21}-\beta_{3} x_{21} x_{25}^{2}\right) \\
& -a_{31}\left(\gamma_{1} x_{33}-\gamma_{2} x_{31}-\gamma_{3} x_{31} x_{35}^{2}\right) \\
& \left.-a_{41}\left(\eta_{1} x_{43}-\eta_{2} x_{41}-\eta_{3} x_{41} x_{45}^{2}\right)-U_{1}\right] \\
+e_{2} & {\left[a_{12}\left(-\alpha_{4} x_{13}+\alpha_{4} x_{14}\right)+a_{22}\left(-\beta_{4} x_{23}+\beta_{4} x_{24}\right)\right.} \\
& -a_{32}\left(-\gamma_{4} x_{33}+\gamma_{4} x_{34}\right) \\
& \left.-a_{42}\left(-\eta_{4} x_{43}+\eta_{4} x_{44}\right)-U_{2}\right] \\
+e_{3} & {\left[a_{13}\left(\alpha_{5} x_{12}-\alpha_{5} x_{11}-\alpha_{6} x_{13}\right)\right.} \\
& +a_{23}\left(\beta_{5} x_{22}-\beta_{5} x_{21}-\beta_{6} x_{23}\right) \\
& -a_{33}\left(\gamma_{5} x_{32}-\gamma_{5} x_{31}-\gamma_{6} x_{33}\right) \\
& \left.-a_{43}\left(\eta_{5} x_{42}-\eta_{5} x_{41}-\eta_{6} x_{43}\right)-U_{3}\right] \\
+e_{4} & {\left[-a_{14} \alpha_{7} x_{12}-a_{24} \beta_{7} x_{22}\right.} \\
& \left.+a_{34} \gamma_{7} x_{32}+a_{44} \eta_{7} x_{42}-U_{4}\right]
\end{aligned}
$$




$$
\begin{aligned}
& +e_{5}\left[a_{15} x_{11}+a_{25} x_{21}-a_{35} x_{31}-a_{45} x_{41}-U_{5}\right] \\
= & e_{1}\left[\Delta_{1}-U_{1}\right]+e_{2}\left[\Delta_{2}-U_{2}\right]+e_{3}\left[\Delta_{3}-U_{3}\right] \\
& +e_{4}\left[\Delta_{4}-U_{4}\right]+e_{5}\left[\Delta_{5}-U_{5}\right]
\end{aligned}
$$

where

$$
\begin{gathered}
\Delta_{1}=a_{11}\left(\alpha_{1} x_{13}-\alpha_{2} x_{11}-\alpha_{3} x_{11} x_{15}^{2}\right) \\
+a_{21}\left(\beta_{1} x_{23}-\beta_{2} x_{21}-\beta_{3} x_{21} x_{25}^{2}\right) \\
-a_{31}\left(\gamma_{1} x_{33}-\gamma_{2} x_{31}-\gamma_{3} x_{31} x_{35}^{2}\right) \\
-a_{41}\left(\eta_{1} x_{43}-\eta_{2} x_{41}-\eta_{3} x_{41} x_{45}^{2}\right), \\
\Delta_{2}=a_{12}\left(-\alpha_{4} x_{13}+\alpha_{4} x_{14}\right)+a_{22}\left(-\beta_{4} x_{23}+\beta_{4} x_{24}\right) \\
-a_{32}\left(-\gamma_{4} x_{33}+\gamma_{4} x_{34}\right)-a_{42}\left(-\eta_{4} x_{43}+\eta_{4} x_{44}\right), \\
\Delta_{3}=a_{13}\left(\alpha_{5} x_{12}-\alpha_{5} x_{11}-\alpha_{6} x_{13}\right) \\
+a_{23}\left(\beta_{5} x_{22}-\beta_{5} x_{21}-\beta_{6} x_{23}\right) \\
-a_{33}\left(\gamma_{5} x_{32}-\gamma_{5} x_{31}-\gamma_{6} x_{33}\right) \\
-a_{43}\left(\eta_{5} x_{42}-\eta_{5} x_{41}-\eta_{6} x_{43}\right), \\
\Delta_{4}=-a_{14} \alpha_{7} x_{12}-a_{24} \beta_{7} x_{22}+a_{34} \gamma_{7} x_{32}+a_{44} \eta_{7} x_{42}, \\
\Delta_{5}=a_{15} x_{11}+a_{25} x_{21}-a_{35} x_{31}-a_{45} x_{41} .
\end{gathered}
$$

On the basis of (18), we have

$$
\begin{aligned}
\Delta_{1}-U_{1}= & -\left(a_{11} x_{11}+a_{21} x_{21}-a_{31} x_{31}-a_{41} x_{41}\right) \\
& -\left(a_{12} x_{12}+a_{22} x_{22}-a_{32} x_{32}-a_{42} x_{42}\right), \\
\Delta_{2}-U_{2}= & \left(a_{11} x_{11}+a_{21} x_{21}-a_{31} x_{31}-a_{41} x_{41}\right) \\
& -\left(a_{12} x_{12}+a_{22} x_{22}-a_{32} x_{32}-a_{42} x_{42}\right) \\
& -\left(a_{13} x_{13}+a_{23} x_{23}-a_{33} x_{33}-a_{43} x_{43}\right), \\
\Delta_{3}-U_{3}= & \left(a_{12} x_{12}+a_{22} x_{22}-a_{32} x_{32}-a_{42} x_{42}\right) \\
& -\left(a_{13} x_{13}+a_{23} x_{23}-a_{33} x_{33}-a_{43} x_{43}\right) \\
& -\left(a_{14} x_{14}+a_{24} x_{24}-a_{34} x_{34}-a_{44} x_{44}\right), \\
\Delta_{4}-U_{4}= & \left(a_{13} x_{13}+a_{23} x_{23}-a_{33} x_{33}-a_{43} x_{43}\right) \\
& -\left(a_{14} x_{14}+a_{24} x_{24}-a_{34} x_{34}-a_{44} x_{44}\right) \\
& -\left(a_{15} x_{15}+a_{25} x_{25}-a_{35} x_{35}-a_{45} x_{45}\right), \\
= & \left(a_{14} x_{14}+a_{24} x_{24}-a_{34} x_{34}-a_{44} x_{44}\right) \\
& -\left(a_{15} x_{15}+a_{25} x_{25}-a_{35} x_{35}-a_{45} x_{45}\right) . \\
\Delta_{5}-U_{5} &
\end{aligned}
$$

Remark 8. For the controller design of combinationcombination synchronization, once we get proper controller (16), then the $u_{i}$ and $u_{i}^{*}(i=1,2,3,4,5)$ in the response systems (10) and (11) can be obtained via $a_{3 i} u_{i}+a_{4 i} u_{i}^{*}=U_{i}$ ( $i=1,2,3,4,5)$. Obviously, these $u_{i}$ and $u_{i}^{*}(i=1,2,3,4,5)$ have wide choices, which can provide the designer with the richness of flexibility. Of course, for the whole controller design of combination-combination synchronization, we merely concern the controller (16).

Next we extend Theorem 6 to other possible cases. 
Corollary 9. If the control law is chosen as follows:

$$
\begin{aligned}
\widetilde{U}_{1}:= & a_{31} u_{1} \\
= & a_{11}\left[\alpha_{1} x_{13}+\left(1-\alpha_{2}\right) x_{11}-\alpha_{3} x_{11} x_{15}^{2}\right] \\
& +a_{21}\left[\beta_{1} x_{23}+\left(1-\beta_{2}\right) x_{21}-\beta_{3} x_{21} x_{25}^{2}\right] \\
& -a_{31}\left[\gamma_{1} x_{33}+\left(1-\gamma_{2}\right) x_{31}-\gamma_{3} x_{31} x_{35}^{2}\right] \\
& +\left(a_{12} x_{12}+a_{22} x_{22}-a_{32} x_{32}\right),
\end{aligned}
$$$$
\widetilde{U}_{2}:=a_{32} u_{2}
$$$$
=a_{12}\left(x_{12}-\alpha_{4} x_{13}+\alpha_{4} x_{14}\right)
$$$$
+a_{22}\left(x_{22}-\beta_{4} x_{23}+\beta_{4} x_{24}\right)
$$$$
-a_{32}\left(x_{32}-\gamma_{4} x_{33}+\gamma_{4} x_{34}\right)
$$$$
-\left(a_{11} x_{11}+a_{21} x_{21}-a_{31} x_{31}\right)
$$$$
+\left(a_{13} x_{13}+a_{23} x_{23}-a_{33} x_{33}\right) \text {, }
$$

$$
\widetilde{U}_{3}:=a_{33} u_{3}
$$$$
=a_{13}\left[\alpha_{5} x_{12}-\alpha_{5} x_{11}+\left(1-\alpha_{6}\right) x_{13}\right]
$$$$
+a_{23}\left[\beta_{5} x_{22}-\beta_{5} x_{21}+\left(1-\beta_{6}\right) x_{23}\right]
$$$$
-a_{33}\left[\gamma_{5} x_{32}-\gamma_{5} x_{31}+\left(1-\gamma_{6}\right) x_{33}\right]
$$$$
-\left(a_{12} x_{12}+a_{22} x_{22}-a_{32} x_{32}\right)
$$$$
+\left(a_{14} x_{14}+a_{24} x_{24}-a_{34} x_{34}\right) \text {, }
$$

$$
\begin{aligned}
\widetilde{U}_{4}:= & a_{34} u_{4} \\
= & -\left(a_{13} x_{13}+a_{23} x_{23}-a_{33} x_{33}\right) \\
& +\left(a_{15} x_{15}+a_{25} x_{25}-a_{35} x_{35}\right) \\
& +a_{14}\left(x_{14}-\alpha_{7} x_{12}\right)+a_{24}\left(x_{24}-\beta_{7} x_{22}\right) \\
& -a_{34}\left(x_{34}-\gamma_{7} x_{32}\right),
\end{aligned}
$$

$$
\begin{aligned}
\widetilde{U}_{5}: & =a_{35} u_{5} \\
= & a_{15}\left(x_{11}+x_{15}\right)+a_{25}\left(x_{21}+x_{25}\right) \\
& -a_{35}\left(x_{31}+x_{35}\right)-\left(a_{14} x_{14}+a_{24} x_{24}-a_{34} x_{34}\right),
\end{aligned}
$$

then the drive systems (4) and (9) will achieve combination synchronization with the response system (10).

Corollary 10. If the control law is chosen as follows:

$$
\begin{aligned}
\widetilde{U}_{1}:= & a_{41} u_{1}^{*} \\
= & a_{11}\left[\alpha_{1} x_{13}+\left(1-\alpha_{2}\right) x_{11}-\alpha_{3} x_{11} x_{15}^{2}\right] \\
& +a_{21}\left[\beta_{1} x_{23}+\left(1-\beta_{2}\right) x_{21}-\beta_{3} x_{21} x_{25}^{2}\right]
\end{aligned}
$$

$$
\begin{aligned}
& -a_{41}\left[\eta_{1} x_{43}+\left(1-\eta_{2}\right) x_{41}-\eta_{3} x_{41} x_{45}^{2}\right] \\
& +\left(a_{12} x_{12}+a_{22} x_{22}-a_{42} x_{42}\right), \\
\widetilde{U}_{2}:= & a_{42} u_{2}^{*} \\
= & a_{12}\left(x_{12}-\alpha_{4} x_{13}+\alpha_{4} x_{14}\right) \\
& +a_{22}\left(x_{22}-\beta_{4} x_{23}+\beta_{4} x_{24}\right) \\
& -a_{42}\left(x_{42}-\eta_{4} x_{43}+\eta_{4} x_{44}\right) \\
& -\left(a_{11} x_{11}+a_{21} x_{21}-a_{41} x_{41}\right) \\
& +\left(a_{13} x_{13}+a_{23} x_{23}-a_{43} x_{43}\right), \\
\widetilde{U}_{3}:= & a_{43} u_{3}^{*} \\
= & a_{13}\left[\alpha_{5} x_{12}-\alpha_{5} x_{11}+\left(1-\alpha_{6}\right) x_{13}\right] \\
& +a_{23}\left[\beta_{5} x_{22}-\beta_{5} x_{21}+\left(1-\beta_{6}\right) x_{23}\right] \\
& -a_{43}\left[\eta_{5} x_{42}-\eta_{5} x_{41}+\left(1-\eta_{6}\right) x_{43}\right] \\
& -\left(a_{12} x_{12}+a_{22} x_{22}-a_{42} x_{42}\right) \\
& +\left(a_{14} x_{14}+a_{24} x_{24}-a_{44} x_{44}\right), \\
\widetilde{U}_{4}:= & a_{44} u_{4}^{*} \\
= & -\left(a_{13} x_{13}+a_{23} x_{23}-a_{43} x_{43}\right) \\
& +\left(a_{15} x_{15}+a_{25} x_{25}-a_{45} x_{45}\right) \\
& +a_{14}\left(x_{14}-\alpha_{7} x_{12}\right)+a_{24}\left(x_{24}-\beta_{7} x_{22}\right) \\
& -a_{44}\left(x_{44}-\eta_{7} x_{42}\right), \\
\widetilde{U}_{5}:= & a_{45} u_{5}^{*} \\
= & a_{15}\left(x_{11}+x_{15}\right)+a_{25}\left(x_{21}+x_{25}\right) \\
& -a_{45}\left(x_{41}+x_{45}\right)-\left(a_{14} x_{14}+a_{24} x_{24}-a_{44} x_{44}\right), \\
&
\end{aligned}
$$

then the drive systems (4) and (9) will achieve combination synchronization with the response system (11).

Corollary 11. If the control law is chosen as follows:

$$
\begin{aligned}
\widetilde{U}_{1}:= & a_{31} u_{1} \\
= & a_{11}\left[\alpha_{1} x_{13}+\left(1-\alpha_{2}\right) x_{11}-\alpha_{3} x_{11} x_{15}^{2}\right] \\
& -a_{31}\left[\gamma_{1} x_{33}+\left(1-\gamma_{2}\right) x_{31}-\gamma_{3} x_{31} x_{35}^{2}\right] \\
& +\left(a_{12} x_{12}-a_{32} x_{32}\right), \\
\widetilde{U}_{2}:= & a_{32} u_{2} \\
= & a_{12}\left(x_{12}-\alpha_{4} x_{13}+\alpha_{4} x_{14}\right)
\end{aligned}
$$




$$
\begin{aligned}
& -a_{32}\left(x_{32}-\gamma_{4} x_{33}+\gamma_{4} x_{34}\right) \\
& -\left(a_{11} x_{11}-a_{31} x_{31}\right)+\left(a_{13} x_{13}-a_{33} x_{33}\right), \\
\widetilde{U}_{3}:= & a_{33} u_{3} \\
= & a_{13}\left[\alpha_{5} x_{12}-\alpha_{5} x_{11}+\left(1-\alpha_{6}\right) x_{13}\right] \\
& -a_{33}\left[\gamma_{5} x_{32}-\gamma_{5} x_{31}+\left(1-\gamma_{6}\right) x_{33}\right] \\
& -\left(a_{12} x_{12}-a_{32} x_{32}\right)+\left(a_{14} x_{14}-a_{34} x_{34}\right), \\
\widetilde{U}_{4}:= & a_{34} u_{4} \\
= & -\left(a_{13} x_{13}-a_{33} x_{33}\right)+a_{14}\left(x_{14}-\alpha_{7} x_{12}\right) \\
& -a_{34}\left(x_{34}-\gamma_{7} x_{32}\right)+\left(a_{15} x_{15}-a_{35} x_{35}\right), \\
\widetilde{U}_{5}:= & a_{35} u_{5} \\
= & a_{15}\left(x_{11}+x_{15}\right)-a_{35}\left(x_{31}+x_{35}\right) \\
& -\left(a_{14} x_{14}-a_{34} x_{34}\right),
\end{aligned}
$$

then the drive system (4) will achieve complete synchronization with the response system (10).

According to Corollary 11, on the complete synchronization criteria between (4) and (11), or (9) and (10), or (9) and (11), one can make some parallel promotions. So we will not repeat all of those corollaries here.

Corollary 12. If the control law is chosen as follows:

$$
\begin{aligned}
\widetilde{U}_{1}:= & a_{31} u_{1} \\
= & -a_{31}\left[\gamma_{1} x_{33}+\left(1-\gamma_{2}\right) x_{31}-\gamma_{3} x_{31} x_{35}^{2}\right] \\
& -a_{32} x_{32}, \\
\widetilde{U}_{2}:= & a_{32} u_{2} \\
= & -a_{32}\left(x_{32}-\gamma_{4} x_{33}+\gamma_{4} x_{34}\right) \\
& +a_{31} x_{31}-a_{33} x_{33}, \\
\widetilde{U}_{3}:= & a_{33} u_{3} \\
= & -a_{33}\left[\gamma_{5} x_{32}-\gamma_{5} x_{31}+\left(1-\gamma_{6}\right) x_{33}\right] \\
& +a_{32} x_{32}-a_{34} x_{34}, \\
\widetilde{U}_{4}:= & a_{34} u_{4} \\
= & a_{33} x_{33}-a_{34}\left(x_{34}-\gamma_{7} x_{32}\right) \\
& -a_{35} x_{35}, \\
\widetilde{U}_{5}:= & a_{35} u_{5} \\
= & -a_{35}\left(x_{31}+x_{35}\right)+a_{34} x_{34},
\end{aligned}
$$

then system (10) is asymptotically stabilizable.
Corollary 13. If the control law is chosen as follows:

$$
\begin{aligned}
\widetilde{U}_{1}:= & a_{41} u_{1}^{*} \\
= & -a_{41}\left[\eta_{1} x_{43}+\left(1-\eta_{2}\right) x_{41}-\eta_{3} x_{41} x_{45}^{2}\right] \\
& -a_{42} x_{42}, \\
\widetilde{U}_{2}:= & a_{42} u_{2}^{*} \\
= & -a_{42}\left(x_{42}-\eta_{4} x_{43}+\eta_{4} x_{44}\right) \\
& +a_{41} x_{41}-a_{43} x_{43}, \\
\widetilde{U}_{3}:= & a_{43} u_{3}^{*} \\
= & -a_{43}\left[\eta_{5} x_{42}-\eta_{5} x_{41}+\left(1-\eta_{6}\right) x_{43}\right] \\
& +a_{42} x_{42}-a_{44} x_{44}, \\
\widetilde{U}_{4}:= & a_{44} u_{4}^{*} \\
= & a_{43} x_{43}-a_{44}\left(x_{44}-\eta_{7} x_{42}\right) \\
& -a_{45} x_{45}, \\
\widetilde{U}_{5}:= & a_{45} u_{5}^{*} \\
= & -a_{45}\left(x_{41}+x_{45}\right)+a_{44} x_{44},
\end{aligned}
$$

then system (11) is asymptotically stabilizable.

Remark 14. Just like Remark 7, the nonlinear degree of control input in the above corollaries is somewhat high. How to design some less conservative criteria, this issue, will be the topic of future research.

Remark 15. In many existing works, the combination of multiple drive systems may induce the combination states to be asymptotically stable or emanative. And theoretically, a direct consequence of such combination of multiple drive systems is the information signal extraction which is extremely difficult or completely useless. In this paper, the combination of two drive systems can be always hyperchaotic; thus, the dynamic behavior is more abundant and complex (see the subsequent numerical example). In theory, when it is used to chaotic communication, this would greatly enhance the security and reliability for high-performance communication.

Remark 16. Theoretically, in the past chaotic communication via drive-response model, the transmitted signal only depends on one drive system. However, in theory, when designing secure communication via combinationcombination synchronization, the transmitted signal and received signal can both be split into multiple different chaotic systems. Compared to some conventional design methods, this design approach maybe have stronger antiattack ability.

In the following, we give a numerical example to illustrate the superiority of theoretical results via computer simulations. 
Assume $\alpha_{1}=\beta_{1}=\gamma_{1}=\eta_{1}=9, \alpha_{2}=\beta_{2}=$ $\gamma_{2}=\eta_{2}=-10.8, \alpha_{3}=\beta_{3}=\gamma_{3}=\eta_{3}=10.8, \alpha_{4}=$ $\beta_{4}=\gamma_{4}=\eta_{4}=1, \alpha_{5}=\beta_{5}=\gamma_{5}=\eta_{5}=30$, $\alpha_{6}=\beta_{6}=\gamma_{6}=\eta_{6}=30, \alpha_{7}=\beta_{7}=\gamma_{7}=\eta_{7}=$ 15. Let $A_{1}=\operatorname{diag}\left(a_{11}, a_{12}, a_{13}, a_{14}, a_{15}\right)=\operatorname{diag}(1,1,1,1,1)$, $A_{2}=\operatorname{diag}\left(a_{21}, a_{22}, a_{23}, a_{24}, a_{25}\right)=\operatorname{diag}(1,1,1,1,1)$, $A_{3}=\operatorname{diag}\left(a_{31}, a_{32}, a_{33}, a_{34}, a_{35}\right)=\operatorname{diag}(1,1,1,1,1), A_{4}=$ $\operatorname{diag}\left(a_{41}, a_{42}, a_{43}, a_{44}, a_{45}\right)=\operatorname{diag}(1,1,1,1,1)$, according to Theorem 6; then the control law can be designed as

$$
\begin{aligned}
& U_{1}=\left[9 x_{13}+11.8 x_{11}-10.8 x_{11} x_{15}^{2}\right] \\
& +\left[9 x_{23}+11.8 x_{21}-10.8 x_{21} x_{25}^{2}\right] \\
& -\left[9 x_{33}+11.8 x_{31}-10.8 x_{31} x_{35}^{2}\right] \\
& -\left[9 x_{43}+11.8 x_{41}-10.8 x_{41} x_{45}^{2}\right] \\
& +\left(x_{12}+x_{22}-x_{32}-x_{42}\right) \text {, } \\
& U_{2}=\left(x_{12}-x_{13}+x_{14}\right)+\left(x_{22}-x_{23}+x_{24}\right) \\
& -\left(x_{32}-x_{33}+x_{34}\right)-\left(x_{42}-x_{43}+x_{44}\right) \\
& -\left(x_{11}+x_{21}-x_{31}-x_{41}\right) \\
& +\left(x_{13}+x_{23}-x_{33}-x_{43}\right) \text {, } \\
& U_{3}=\left[30 x_{12}-30 x_{11}-29 x_{13}\right] \\
& +\left[30 x_{22}-30 x_{21}-29 x_{23}\right] \\
& -\left[30 x_{32}-30 x_{31}-29 x_{33}\right] \\
& -\left[30 x_{42}-30 x_{41}-29 x_{43}\right] \\
& -\left(x_{12}+x_{22}-x_{32}-x_{42}\right) \\
& +\left(x_{14}+x_{24}-x_{34}-x_{44}\right) \text {, } \\
& U_{4}=-\left(x_{13}+x_{23}-x_{33}-x_{43}\right) \\
& +\left(x_{14}-15 x_{12}\right)+\left(x_{24}-15 x_{22}\right) \\
& -\left(x_{34}-15 x_{32}\right)-\left(x_{44}-15 x_{42}\right) \\
& +\left(x_{15}+x_{25}-x_{35}-x_{45}\right) \text {, } \\
& U_{5}=x_{11}+x_{15}+x_{21}+x_{25} \\
& -\left(x_{31}+x_{35}\right)-\left(x_{41}+x_{45}\right) \\
& -\left(x_{14}+x_{24}-x_{34}-x_{44}\right) \text {. }
\end{aligned}
$$

By (16), it follows that

$$
\begin{aligned}
& U_{1}=u_{1}+u_{1}^{*}, \\
& U_{2}=u_{2}+u_{2}^{*}, \\
& U_{3}=u_{3}+u_{3}^{*},
\end{aligned}
$$

$$
\begin{aligned}
& U_{4}=u_{4}+u_{4}^{*}, \\
& U_{5}=u_{5}+u_{5}^{*} .
\end{aligned}
$$

then $u_{i}$ and $u_{i}^{*}(i=1,2,3,4,5)$ can be chosen according to different requirements. For example, it allows

$$
\begin{aligned}
& u_{1}=\left[9 x_{13}+11.8 x_{11}-10.8 x_{11} x_{15}^{2}\right] \\
& +\left[9 x_{23}+11.8 x_{21}-10.8 x_{21} x_{25}^{2}\right] \\
& -\left[9 x_{33}+11.8 x_{31}-10.8 x_{31} x_{35}^{2}\right] \\
& -\left[9 x_{43}+11.8 x_{41}-10.8 x_{41} x_{45}^{2}\right] \text {, } \\
& u_{1}^{*}=x_{12}+x_{22}-x_{32}-x_{42} \text {, } \\
& u_{2}=\left(x_{12}-x_{13}+x_{14}\right)+\left(x_{22}-x_{23}+x_{24}\right) \\
& -\left(x_{32}-x_{33}+x_{34}\right)-\left(x_{42}-x_{43}+x_{44}\right) \text {, } \\
& u_{2}^{*}=-\left(x_{11}+x_{21}-x_{31}-x_{41}\right) \\
& +\left(x_{13}+x_{23}-x_{33}-x_{43}\right) \text {, } \\
& u_{3}=\left[30 x_{12}-30 x_{11}-29 x_{13}\right] \\
& +\left[30 x_{22}-30 x_{21}-29 x_{23}\right] \\
& -\left[30 x_{32}-30 x_{31}-29 x_{33}\right] \\
& -\left[30 x_{42}-30 x_{41}-29 x_{43}\right] \text {, } \\
& u_{3}^{*}=-\left(x_{12}+x_{22}-x_{32}-x_{42}\right) \\
& +\left(x_{14}+x_{24}-x_{34}-x_{44}\right), \\
& u_{4}=-\left(x_{13}+x_{23}-x_{33}-x_{43}\right) \\
& +\left(x_{14}-15 x_{12}\right)+\left(x_{24}-15 x_{22}\right) \\
& -\left(x_{34}-15 x_{32}\right)-\left(x_{44}-15 x_{42}\right) \text {, } \\
& u_{4}^{*}=x_{15}+x_{25}-x_{35}-x_{45} \text {, } \\
& u_{5}=x_{11}+x_{15}+x_{21}+x_{25} \\
& -\left(x_{31}+x_{35}\right)-\left(x_{41}+x_{45}\right), \\
& u_{5}^{*}=-x_{14}+x_{24}-x_{34}-x_{44} \text {. }
\end{aligned}
$$

Of course, the $u_{i}$ and $u_{i}^{*}(i=1,2,3,4,5)$ in (33) are just one case rather than the norm. The designers can choose what they need via the combination-combination controller (31).

Simulation result of the combination of two drive systems (4) and (9) is depicted in Figure 4. The computer simulation suggests that the combination of two drive systems (4) and (9) has a hyperchaotic attractor, as shown in Figure 4, which has verified that the combination of drive systems (4) and (9) remains hyperchaotic. Meanwhile, according to Theorem 6, the combination of two drive systems (4) and (9) can achieve 
Memristor attractor: 3D projection

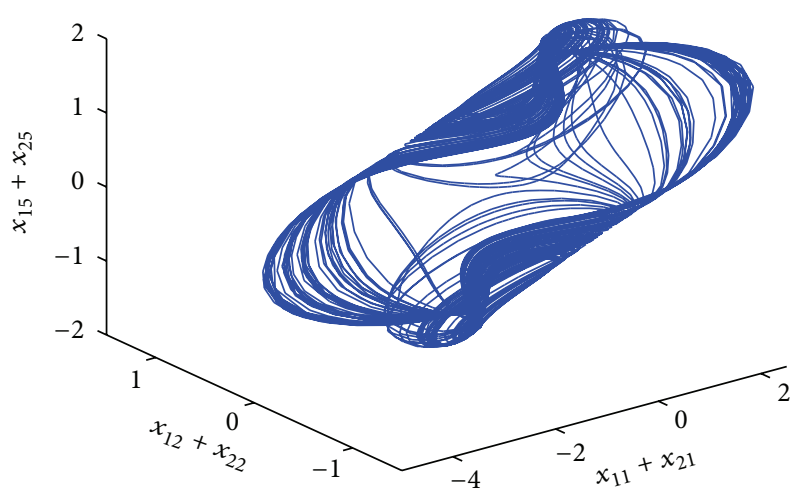

(a)

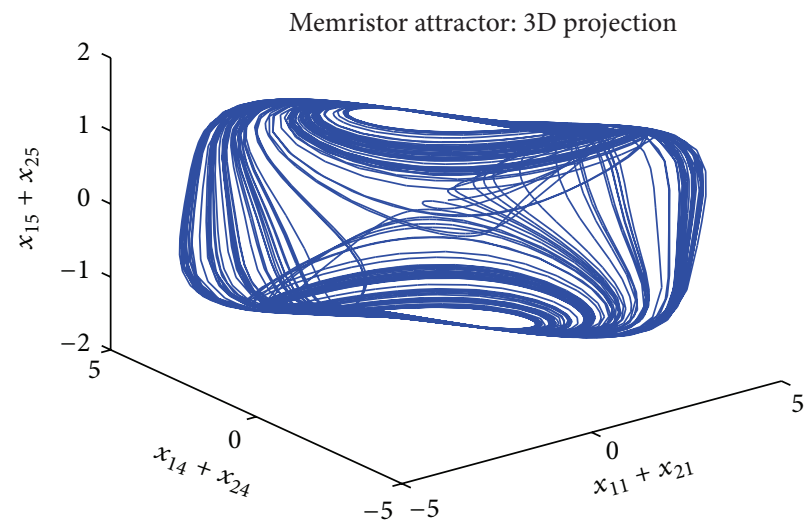

(c)

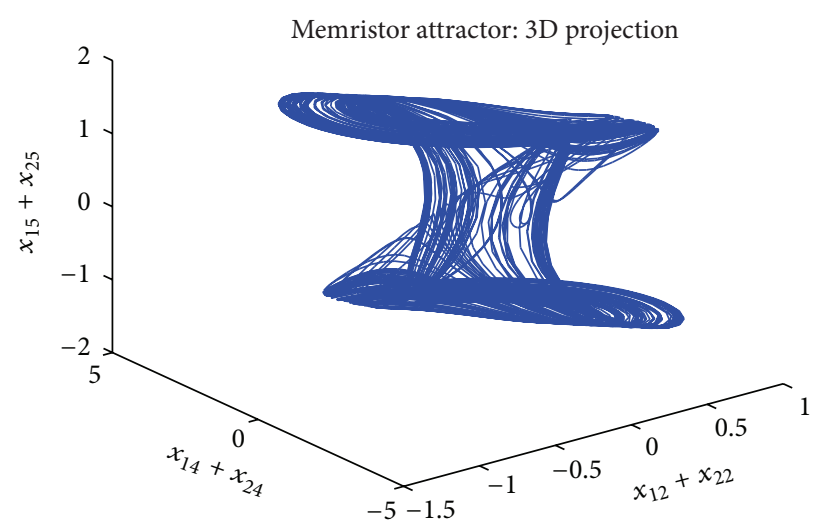

(e)
Memristor attractor: 3D projection

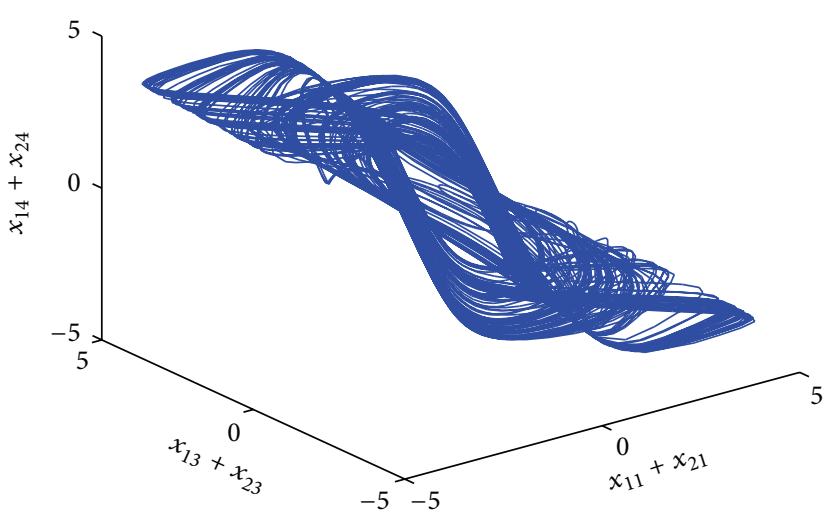

(b)

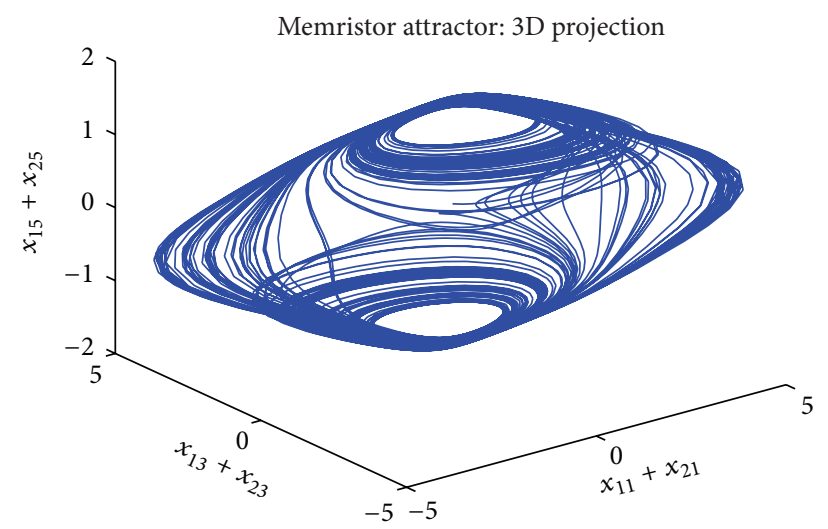

(d)

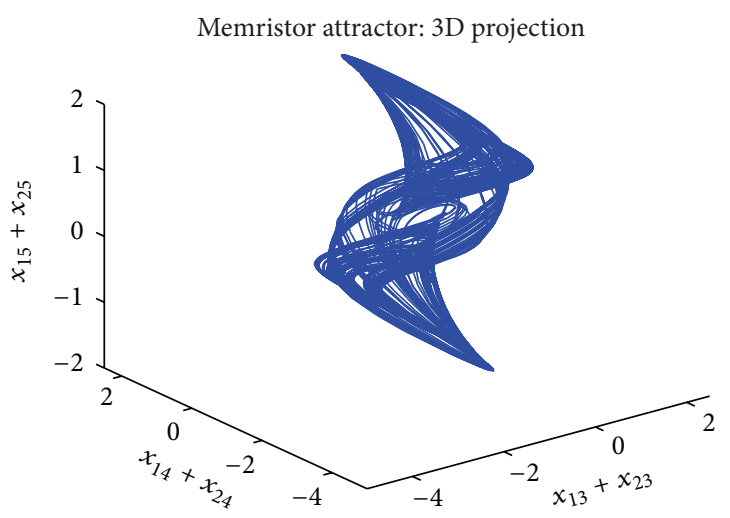

(f)

FIGURE 4: 3D projections of the hyperchaotic attractor from combination of two drive systems.

synchronization with the combination of two response systems (10) and (11). Figure 5 depicts the time response of the synchronization error $e(t)=\left(e_{1}(t), e_{2}(t), e_{3}(t), e_{4}(t), e_{5}(t)\right)^{T}$.

\section{Concluding Remarks}

A fifth-order complex memristor oscillator system with hyperchaotic effect is presented in this paper. Some sufficient conditions are derived to guarantee the hyperchaotic complex memristor oscillator system for realizing combinationcombination synchronization. The hyperchaotic complex memristor oscillator system is useful in secure communication systems with enhanced security features that protect against deciphering. Also, the scheme of combinationcombination synchronization might overcome some design issues associated with previous schemes for using chaos in communications. Application of new intelligent control 


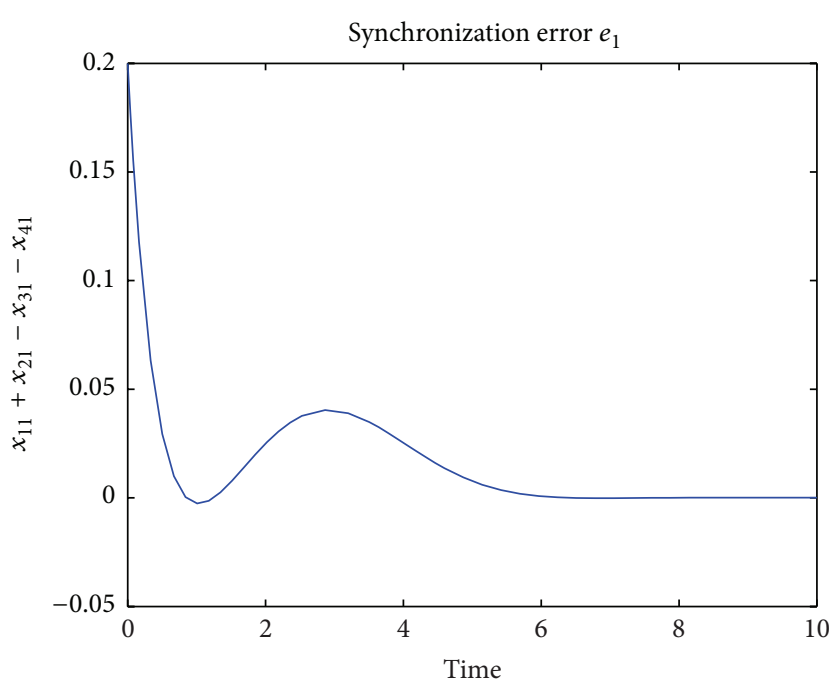

(a)

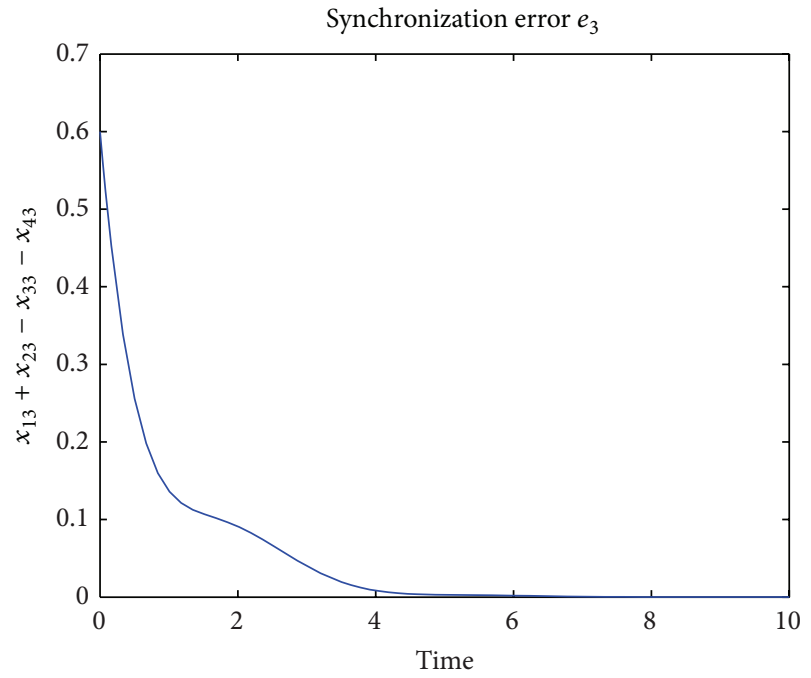

(c)

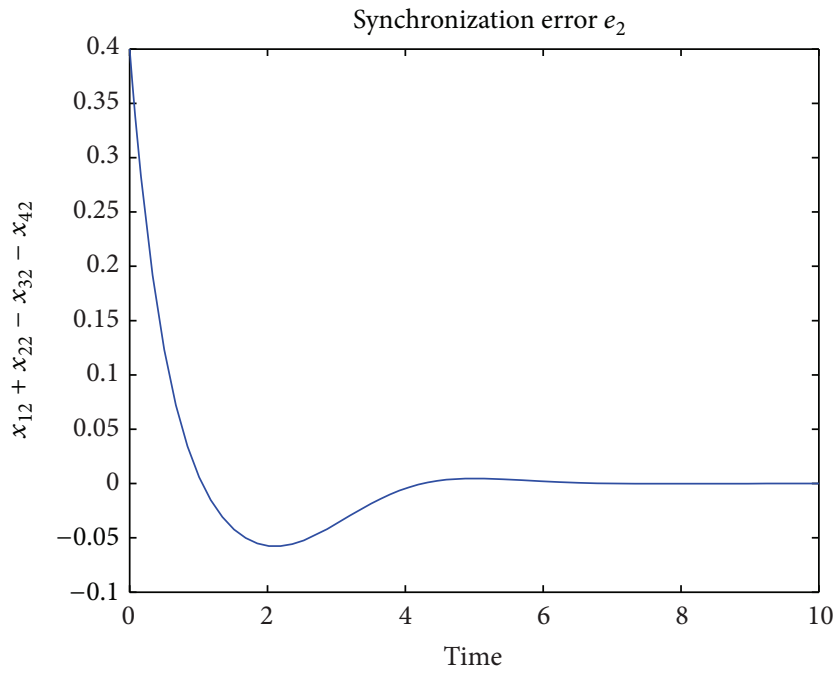

(b)

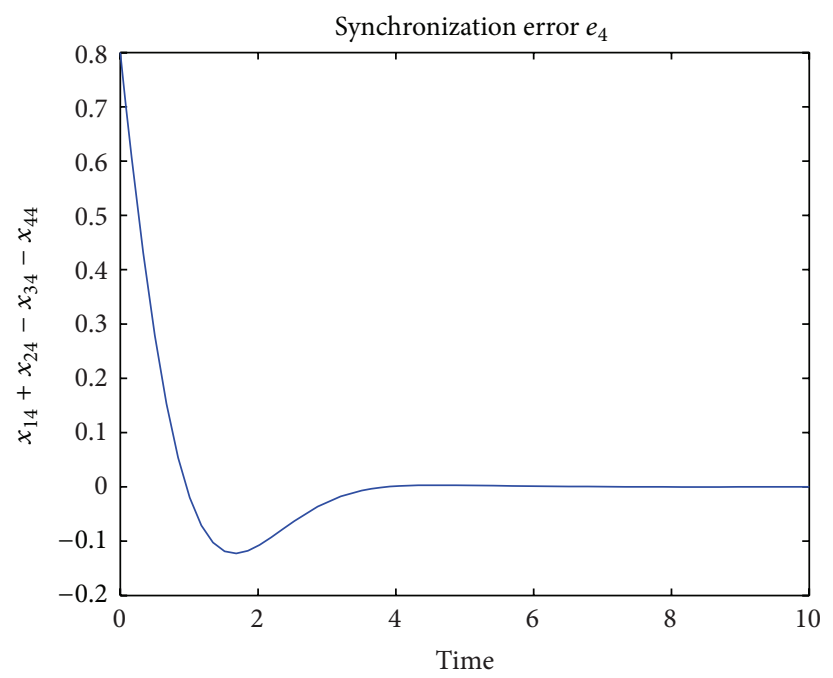

(d)

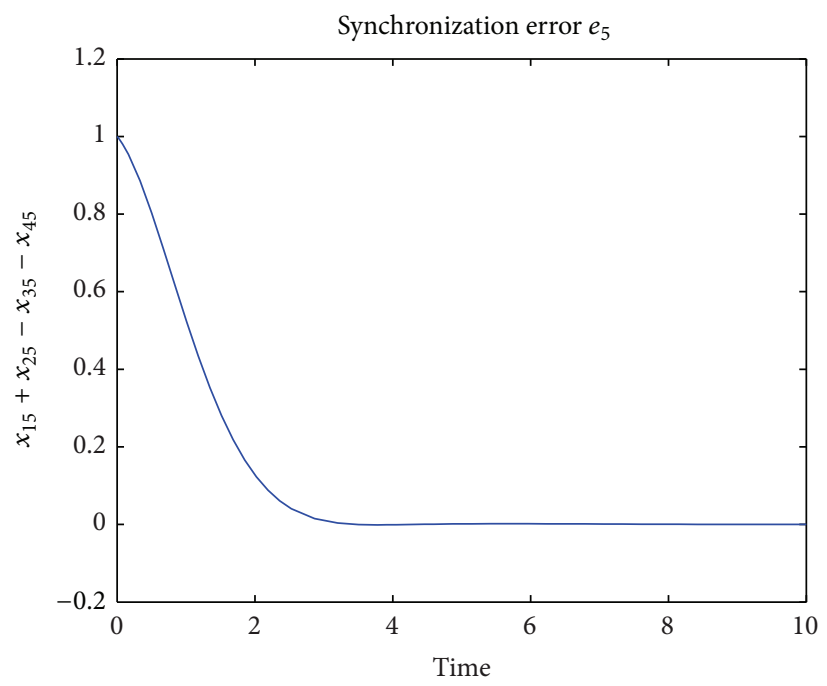

(e)

FIGURE 5: Time response curve for synchronization error $e(t)=\left(e_{1}(t), e_{2}(t), e_{3}(t), e_{4}(t), e_{5}(t)\right)^{T}$. 
methods to complex memristor oscillator model yields a deep insight into the behaviors under investigation.

\section{Conflict of Interests}

The author declares that there is no conflict of interests regarding the publication of this paper.

\section{Acknowledgment}

The work is supported by the Research Project of Hubei Provincial Department of Education of China under Grant T201412.

\section{References}

[1] B. C. Bao, Z. Liu, and J. P. Xu, "Steady periodic Memristor oscillator with transient chaotic behaviours," Electronics Letters, vol. 46, no. 3, pp. 237-238, 2010.

[2] F. Corinto, A. Ascoli, and M. Gilli, "Nonlinear dynamics of Memristor oscillators," IEEE Transactions on Circuits and Systems I: Regular Papers, vol. 58, no. 6, pp. 1323-1336, 2011.

[3] M. Itoh and L. O. Chua, "Memristor oscillators," International Journal of Bifurcation and Chaos, vol. 18, no. 11, pp. 3183-3206, 2008.

[4] B. Muthuswamy and P. P. Kokate, "Memristor-based chaotic circuits," IETE Technical Review, vol. 26, no. 6, pp. 417-429, 2009.

[5] R. Riaza, "First order mem-circuits: modeling, nonlinear oscillations and bifurcations," IEEE Transactions on Circuits and Systems I: Regular Papers, vol. 60, no. 6, pp. 1570-1583, 2013.

[6] A. Talukdar, A. G. Radwan, and K. N. Salama, "Generalized model for Memristor-based Wien family oscillators," Microelectronics Journal, vol. 42, no. 9, pp. 1032-1038, 2011.

[7] A. Talukdar, A. G. Radwan, and K. N. Salama, "Non linear dynamics of memristor based 3rd order oscillatory system," Microelectronics Journal, vol. 43, no. 3, pp. 169-175, 2012.

[8] S. P. Wen, G. Bao, Z. G. Zeng, Y. R. Chen, and T. W. Huang, "Global exponential synchronization of Memristorbased recurrent neural networks with time-varying delays," Neural Networks, vol. 48, pp. 195-203, 2013.

[9] A. L. Wu, "Hyperchaos synchronization of Memristor oscillator system via combination scheme," Advances in Difference Equations, vol. 2014, article 86, pp. 1-11, 2014.

[10] A. L. Wu and J. Zhang, "Compound synchronization of fourthorder Memristor oscillator," Advances in Difference Equations, vol. 2014, article 100, pp. 1-16, 2014.

[11] A. L. Wu, S. P. Wen, and Z. G. Zeng, "Synchronization control of a class of Memristor-based recurrent neural networks," Information Sciences, vol. 183, no. 1, pp. 106-116, 2012.

[12] A. L. Wu and Z. G. Zeng, "Dynamic behaviors of Memristorbased recurrent neural networks with time-varying delays," Neural Networks, vol. 36, pp. 1-10, 2012.

[13] A. L. Wu and Z. G. Zeng, "Exponential stabilization of memristive neural networks with time delays," IEEE Transactions on Neural Networks and Learning Systems, vol. 23, no. 12, pp. 19191929, 2012.

[14] A. L. Wu, Z. G. Zeng, and J. Xiao, "Dynamic evolution evoked by external inputs in Memristor-based wavelet neural networks with different memductance functions," Advances in Difference Equations, vol. 2013, article 258, pp. 1-14, 2013.

[15] T. W. Huang, C. D. Li, W. W. Yu, and G. R. Chen, "Synchronization of delayed chaotic systems with parameter mismatches by using intermittent linear state feedback," Nonlinearity, vol. 22, no. 3, pp. 569-584, 2009.

[16] T. W. Huang and C. D. Li, "Chaotic synchronization by the intermittent feedback method," Journal of Computational and Applied Mathematics, vol. 234, no. 4, pp. 1097-1104, 2010.

[17] T. W. Huang, G. R. Chen, and J. Kurths, "Synchronization of chaotic systems with time-varying coupling delays," Discrete and Continuous Dynamical Systems B, vol. 16, no. 4, pp. 10711082, 2011.

[18] T. W. Huang, D. Gao, C. D. Li, and M. Q. Xiao, "Anticipating synchronization through optimal feedback control," Journal of Global Optimization, vol. 52, no. 2, pp. 281-290, 2012.

[19] C. D. Li and X. F. Liao, "Anti-synchronization of a class of coupled chaotic systems via linear feedback control," International Journal of Bifurcation and Chaos, vol. 16, no. 4, pp. 1041-1047, 2006.

[20] A. L. Wu and Z. G. Zeng, "Anti-synchronization control of a class of memristive recurrent neural networks," Communications in Nonlinear Science and Numerical Simulation, vol. 18, no. 2, pp. 373-385, 2013.

[21] G. D. Zhang, Y. Shen, and L. M. Wang, "Global antisynchronization of a class of chaotic memristive neural networks with time-varying delays," Neural Networks, vol. 46, pp. $1-8,2013$.

[22] D. Li, X. L. Li, D. Cui, and Z. H. Li, "Phase synchronization with harmonic wavelet transform with application to neuronal populations," Neurocomputing, vol. 74, no. 17, pp. 3389-3403, 2011.

[23] Z. Odibat, "A note on phase synchronization in coupled chaotic fractional order systems," Nonlinear Analysis: Real World Applications, vol. 13, no. 2, pp. 779-789, 2012.

[24] C. D. Li, X. F. Liao, and K.-W. Wong, "Chaotic lag synchronization of coupled time-delayed systems and its applications in secure communication," Physica D: Nonlinear Phenomena, vol. 194, no. 3-4, pp. 187-202, 2004.

[25] X. S. Yang, Q. X. Zhu, and C. X. Huang, "Generalized lagsynchronization of chaotic mix-delayed systems with uncertain parameters and unknown perturbations," Nonlinear Analysis: Real World Applications, vol. 12, no. 1, pp. 93-105, 2011.

[26] J. Yu, C. Hu, H. J. Jiang, and Z. D. Teng, "Exponential lag synchronization for delayed fuzzy cellular neural networks via periodically intermittent control," Mathematics and Computers in Simulation, vol. 82, no. 5, pp. 895-908, 2012.

[27] J.-W. Xiao, Z.-W. Wang, W.-T. Miao, and Y.-W. Wang, "Adaptive pinning control for the projective synchronization of driveresponse dynamical networks," Applied Mathematics and Computation, vol. 219, no. 5, pp. 2780-2788, 2012.

[28] J. W. Sun, Y. Shen, G. D. Zhang, C. J. Xu, and G. Z. Cui, "Combination-combination synchronization among four identical or different chaotic systems," Nonlinear Dynamics, vol. 73, no. 3, pp. 1211-1222, 2013. 


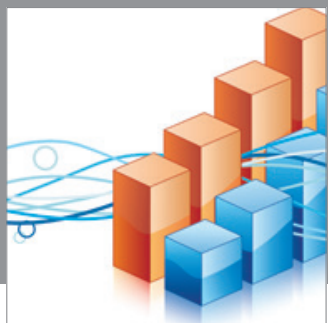

Advances in

Operations Research

mansans

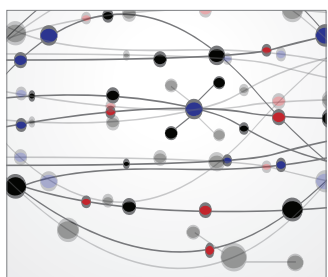

The Scientific World Journal
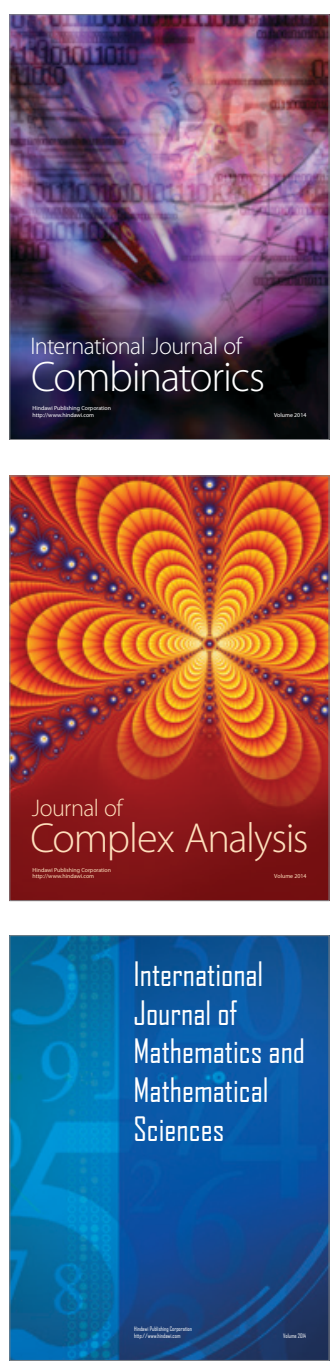
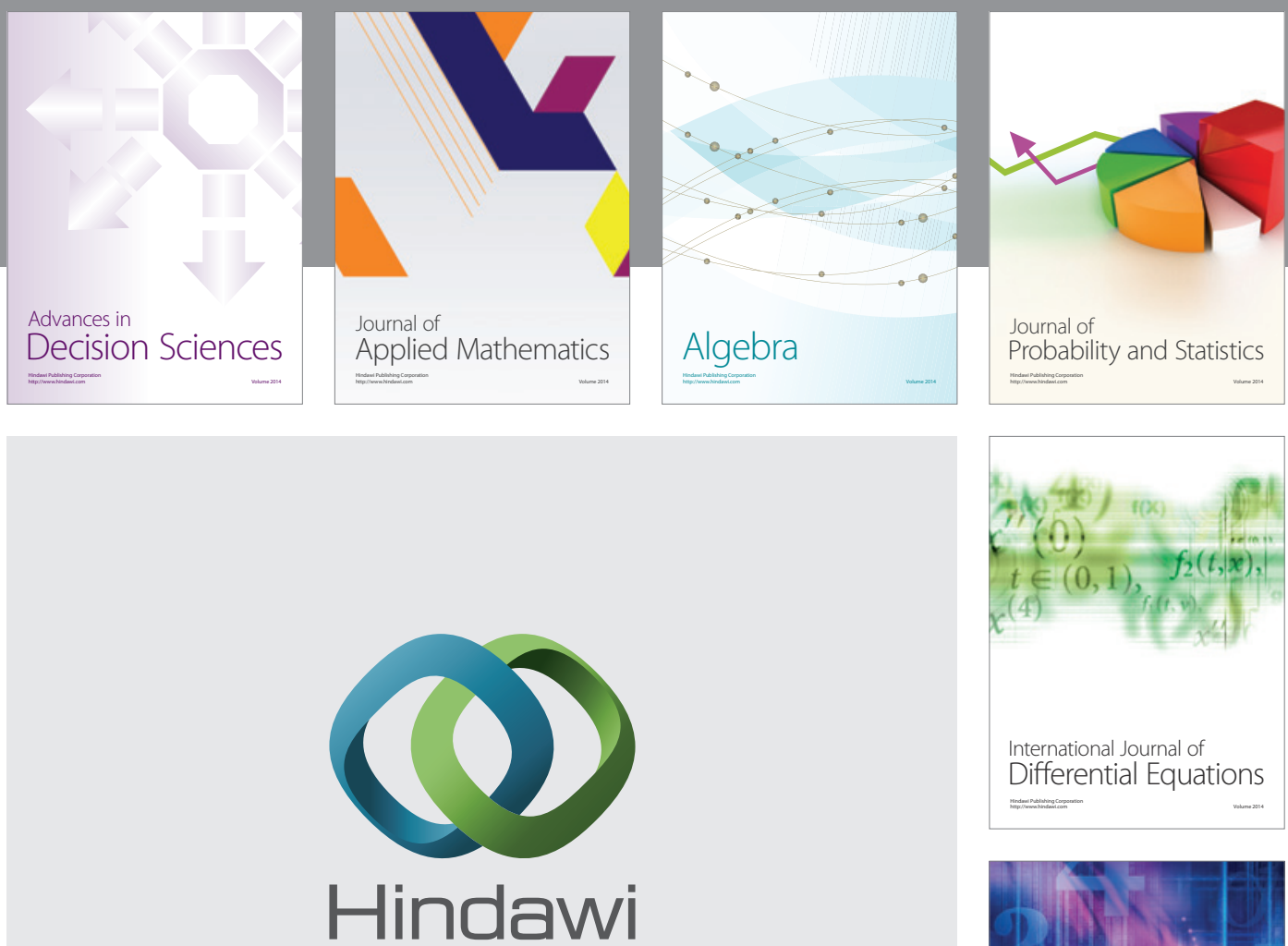

Submit your manuscripts at http://www.hindawi.com
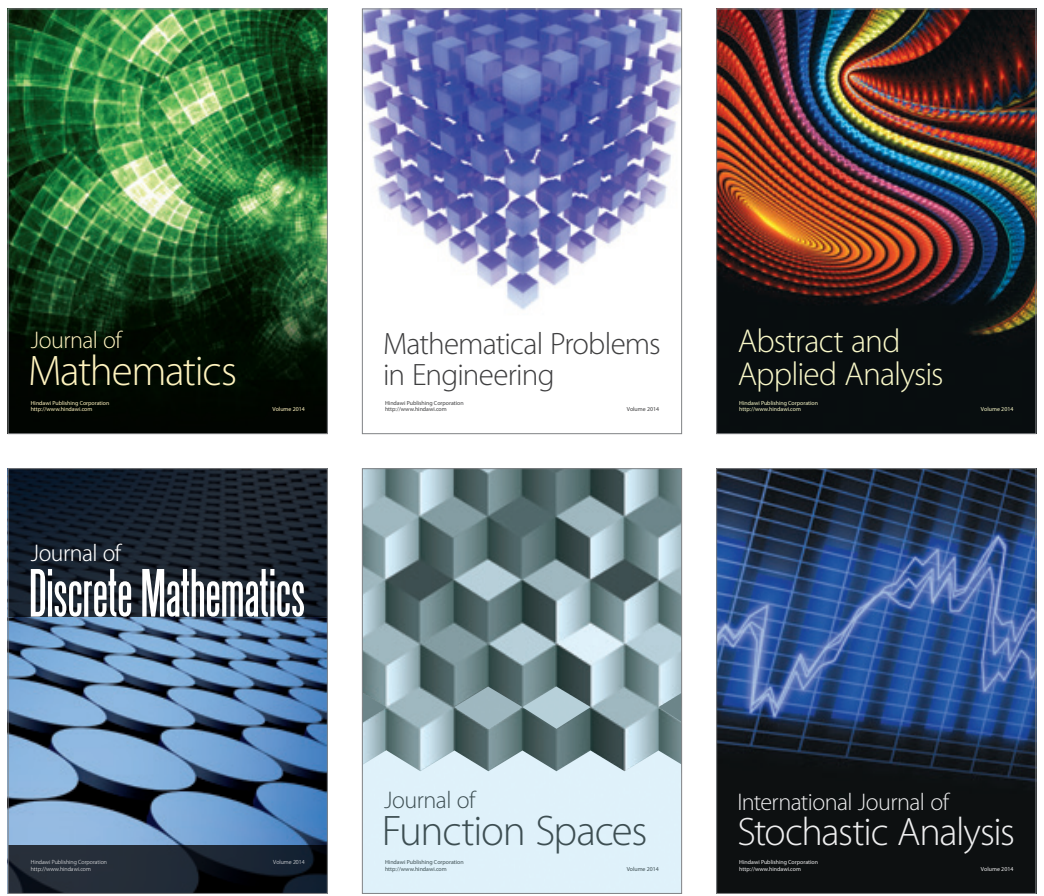

Journal of

Function Spaces

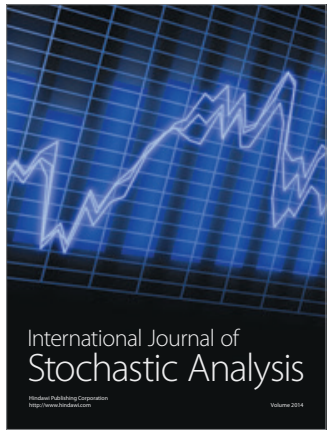

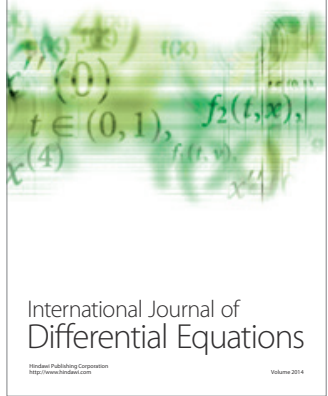
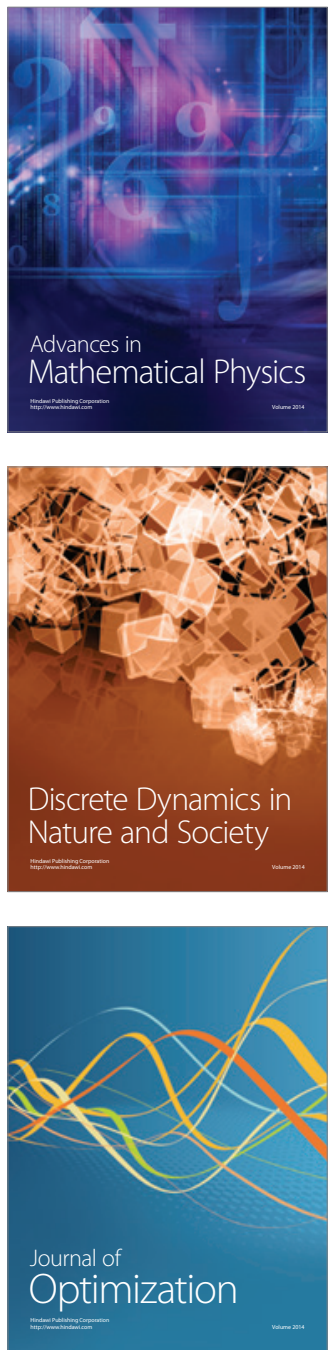